\title{
ENDOGAMIA EN EL MAÍZ EN LA SELECCIÓN RECURRENTE PARA APTITUD COMBINATORIA
}

\section{INBREEDING IN MAIZE SUBMITTED TO RECURRENT SELECTION FOR COMBINING ABILITY}

\author{
Fidel Márquez-Sánchez ${ }^{\dagger}$
}

Centro Regional Universitario Occidente, Universidad Autónoma Chapingo. Rosario Castellanos 2332, Col. Residencial la Cruz. 44950, Guadalajara, Jalisco, México.

\section{RESUMEN}

En los tres tipos de selección recurrente para aptitud combinatoria en el maíz (Zea mays L.) se usan como unidades de selección a líneas $\mathrm{S}_{1}$, mientras que los criterios de selección son los mestizos hechos por las cruzas entre las unidades de selección y los probadores: una variedad de polinización libre, una línea altamente endogámica y la población B para las líneas $S_{1}$ derivadas de $A$, y la población A para las líneas $S_{1}$ derivadas de $B$. La endogamia que se genera ocurre por los cruzamientos entre las líneas seleccionadas $S_{1}$ en los tres tipos de selección recurrente, aunque las líneas $\mathrm{S}_{1}$ no serán las mismas en los tres tipos de selección. En los cálculos que aquí se presentan se usaron 20 líneas $S_{1}$ y 20 plantas por línea, que produjeron una alta endogamia (0.59) en el ciclo 10 de selección. Para reducir la endogamia a 0.20 basta con hacer solamente dos o tres ciclos de selección.

Palabras clave: Zea mays, endogamia, selección recurrente, aptitud combinatoria.

\section{SUMMARY}

In the three types of recurrent selection for combining ability in maize (Zea mays $\mathrm{L}$.), $\mathrm{S}_{1}$ lines are used as selection units. The selection criteria are the top crosses made among $S_{1}$ lines and their respective testers: an open-pollinated variety; a highly homogeneous line; and variety $B$ for the $S_{1}$ lines from variety $A$, and variety $A$ for $S_{1}$ lines from variety $B$. Generated inbreeding occurs because of crossing among selected $S_{1}$ lines in the three types of recurrent selection, though selected $S_{1}$ lines are not the same in the three types of selection. In this paper, considering 20 lines $S_{1}$ y 20 plants per line, inbreeding in cycle ten reached a very high value $(0.59)$. Reduction of inbreeding to 0.20 can be achieved by making only two or three selection cycles.

Index words: Zea mays, inbreeding, recurrent selection, combining ability.

\section{INTRODUCCIÓN}

En los ciclos de selección recurrente aplicada al maíz (Zea mays L.) se presenta endogamia debido a que el número de familias seleccionadas es relativamente pequeño, aún sin considerar el ajuste por el número efectivo de varianza que participa en el cálculo del coeficiente de endogamia. Para reducir el efecto nocivo de la endogamia, o bien se incrementa el número de familias o se incrementa la presión de selección, pero la segunda opción causará una reducción del número de familias por probar.
En el cálculo de la endogamia que ocurre en la selección familiar aplicada al maíz, Márquez-Sánchez (2009) usó 180 familias con selección de las mejores 10 en cada ciclo. Posteriormente, Márquez-Sánchez (2013) propuso aumentar a 20 el número de familias $(n=20)$ con 20 plantas por familia $(m=20)$, para un total de 400 plantas.

Para la selección recurrente de líneas $\mathrm{S}_{1}$, de familias de hermanos completos y de familias de medios hermanos, Márquez-Sánchez (2009) calculó el número efectivo de varianza de acuerdo con Crossa y Venkovsky (1997), y para estos tres tipos de selección obtuvo que los números de familias por probar son iguales a 9,6 y 7 , respectivamente. Aquí es de destacar la notable contradicción de que el número de líneas en las familias de hermanos completos resulta menor que el número en las familias de medios hermanos.

La finalidad del presente escrito es calcular la endogamia al incrementar el número de familias bajo prueba en la selección recurrente para aptitud combinatoria $(\mathrm{AC})$, con la presión de selección acostumbrada y con 400 plantas.

\section{MATERIALES Y MÉTODOS}

Los cálculos en este apartado pueden aplicarse a los tipos de selección recurrente para: aptitud combinatoria general (ACG), aptitud combinatoria específica (ACE) y la selección recíproca recurrente (SRR) para dos variedades (A y B), pues aunque el cálculo de la endogamia es el mismo para los tres tipos de familias, las líneas seleccionadas son diferentes debido a los tipos de probadores que se usan en los tres tipos de selección recurrente: variedad de polinización libre para ACG; línea pura para ACE; y en la SRR la variedad A para la variedad B, y la variedad B para la variedad A.

El cálculo de la endogamia se hizo de acuerdo con el número de líneas $S_{1}$ seleccionadas, antes de la obtención de nuevas líneas $S_{1}$ para el siguiente ciclo de selección. El cálculo depende del número efectivo de varianza $\left[N_{e(v)}\right]$ el cual se obtendrá de acuerdo con la intensidad de selección 
$(s)$, del número total de líneas $(n)$ y del número de plantas por línea $(m)$, según Crossa y Venkovsky (1997). Con $N_{e(v)}$ se calculan los números ajustados $n^{*}$ y $m^{*}$, con $Q=n / m$. Entonces:

$N=n m=400 ; n=20 y m=20 ; s=20 / 400=0.05, Q=n / m=20 / 20=1$.

$N_{e(v)(S 1)}=N[s /(1+1 / 20]=400[0.05 /(1+1 / 20)]=19.0476$

Por lo que: $n^{*}=\left[N_{e(v)} Q\right]^{1 / 2}=\left(\begin{array}{ll}19.0476 \times 1)^{1 / 2}=4.364357 \\ \hline\end{array}\right.$

$$
m^{*}=\left[N_{e(v)} / Q\right]^{1 / 2}=(19.0476 / 1)^{1 / 2}=4.364357
$$

De modo que: $Q^{*}=n^{*} / m^{*}=1$

La recombinación entre las líneas seleccionadas se hizo conforme al diseño dialélico en un solo sentido. En el Cuadro 1 se muestra el dialélico hecho con $n$ líneas; los cruzamientos se encuentran en la columna; en la diagonal están los cruzamientos dialélicos en número $m(m-1) / 2$; arriba de la diagonal, en cada celda hay $m^{2}$ cruzamientos entre plantas.

Se debe considerar también que en total de $n$ líneas, los cruzamientos entre ellas son: $n m(m-1) / 2$ y $n m^{2}(n-1) / 2$; a su vez, estos números se codifican al dividir a cada uno por $n m / 2$, de manera que quedan como: $m-1$ y $m(n-1)$. Con el objeto de calcular la endogamia en cada tipo de cruzamientos (dentro de las líneas y entre las líneas), es necesario tomar en cuenta a las coancestrías en el ciclo 0 de selección, las que en el ciclo 1 de selección darán lugar a los respectivos valores de la endogamia.

En los cruzamientos dentro de las líneas $S_{1}$ la endogamia es la misma que la de las líneas $S_{1}$ (Márquez-Sánchez, 2007), y los cruzamientos entre las líneas producirán híbridos entre líneas $\mathrm{S}_{1}$ con endogamia igual a $\mathrm{F}_{1}$. En el Cuadro 2 se muestra el total de esta información. Finalmente, la en- dogamia de la recombinación entre las líneas $\mathrm{S}_{1}$ se calculó como el promedio de la suma de los números codificados por las coancestrías, y se divide entre en total de los números codificados $(n m-1)$.

\section{RESULTADOS Y DISCUSIÓN}

Como la población de donde se derivaron las líneas $S_{1}$ corresponde al ciclo 0 , entonces el ciclo 1 corresponde al de las líneas $\mathrm{S}_{1}$, y la endogamia del ciclo 2 sería:

$$
\begin{aligned}
F\left(A C-S_{1}\right)_{2} & =\left[(m-1)(1 / 2)\left(1+F_{0}\right)+m(n-1) F_{1}\right] /(n m-1) \\
& =[1 /(n m-1)]\left[(1 / 2) m+(1 / 2) m F_{0}-1 / 2-(1 / 2) F_{0}+n m F_{1}-m F_{1}\right] \\
& =\left[(1 / 2(n m-1)]\left[(m-1)+(m-1) F_{0}+2 m(n-1) F_{1}\right]\right. \\
& =\left[(1 / 2(n m-1)]\left[(m-1)+2 m(n-1) F_{1}+(m-1) F_{0}\right]\right.
\end{aligned}
$$

Por extrapolación, la ecuación para cualquier ciclo de selección $t$ es:

[Ec. 1]

$F\left(A C-S_{1}\right)_{t}=[1 / 36.095224]\left(3.364357+29.366510 F_{t-1}+3.364357 F_{t-2}\right)$

En la Figura 1 se muestra la curva de endogamia de la Ec. 1 para 100 ciclos de selección. Puede verse que en el ciclo 10 (un número muy usado de ciclos de selección) la endogamia es igual 0.60, valor que es muy alto. Para alcanzar un valor endogámico de alrededor de 0.20 , sólo serían necesarios dos o tres ciclos de selección.

\section{CONCLUSIONES}

La selección recurrente para aptitud combinatoria general es un método que genera valores muy altos de la endogamia en los primeros ciclos de selección.

Cuadro 1. Diseño dialélico en un solo sentido con $n$ líneas.

\begin{tabular}{llccc}
\hline$n$ & 1 & 2 & 3 & $n$ \\
\hline 1 & & 12 & 13 & $1 n$ \\
2 & & 23 & $2 n$ \\
3 & & & $3 n$ \\
\hline
\end{tabular}

Cuadro 2. Información para el cálculo de la endogamia procedente de la recombinación de líneas $\mathrm{S}_{1}$ seleccionadas.

\begin{tabular}{lccc}
\hline Cruzamientos & Números reales & Números codificados & Coancestrías \\
\hline Dentro de celdas & $n m(m-1) / 2$ & $m-1$ & $(1 / 2)\left(1+F_{0}\right)$ \\
Entre celdas & $n m^{2}(n-1) / 2$ & $m(n-1)$ & $F_{1}$ \\
\hline
\end{tabular}

Total de números reales: $n m(m-1) / 2+n m^{2}(n-1) / 2=(n m / 2)(n m-1)$; Total números codificados: $n m-1$. 


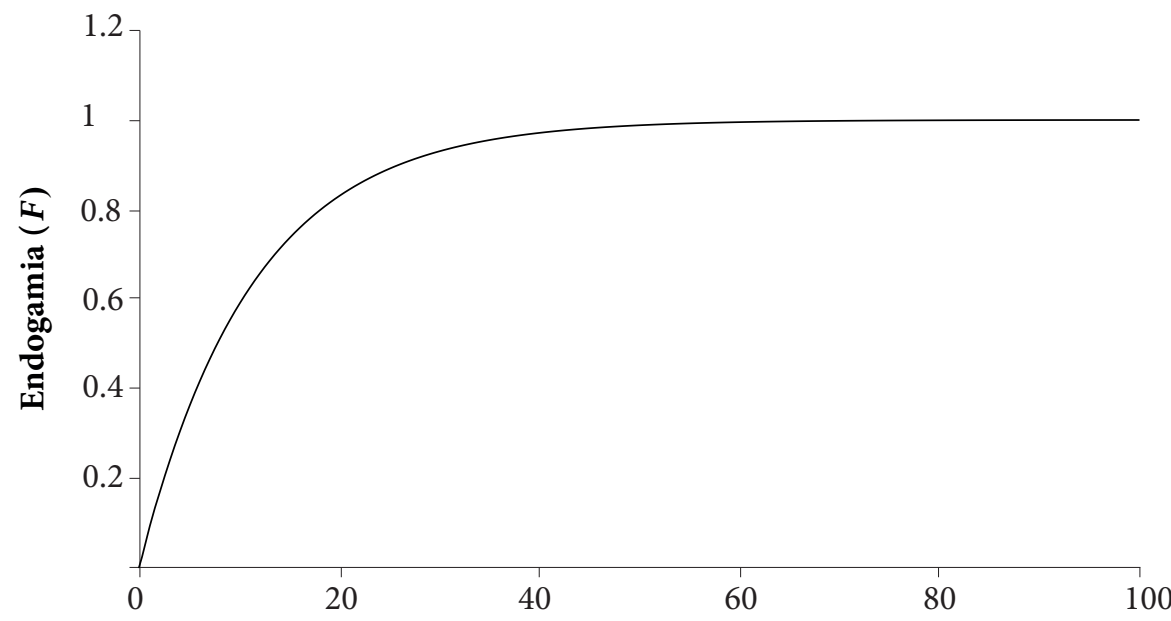

Ciclos de selección $(t)$

Figura 1. Valores de la endogamia en $\mathbf{1 0 0}$ ciclos de selección recurrente para aptitud combinatoria general en maíz, calculados con $F\left(A C-S_{1}\right)_{t}=[1 / 36.095224]\left(3.364357+29.366510 F_{t-1}+3.364357 F_{t-2}\right)$.

\section{BIBLIOGRAFÍA}

Crossa J. and R. Venkovsky (1997) Variance effective population size for two-stage sampling of monoecious species. Crop Science 37:1426.
Márquez-Sánchez F. (2009) Expected inbreeding in recurrent selection in maize. III: Selection in $\mathrm{S}_{1}$ lines and full-sib and half-sib families. Maydica 54:109-111.

Márquez-Sánchez F. (2013) Cálculo de la endogamia en la selección recurrente de familias en el maíz. Revista Mexicana de Ciencias Agrícolas 4:153-158. 